\title{
Acceleration, pressure and related quantities in the proximity of the turbulent/non-turbulent interface
}

\section{Journal Article}

Author(s):

Holzner, Markus; Lüthi, B.; Tsinober, A.; Kinzelbach, Wolfgang

Publication date:

2009-11-25

Permanent link:

https://doi.org/10.3929/ethz-b-000019798

Rights / license:

In Copyright - Non-Commercial Use Permitted

Originally published in:

Journal of Fluid Mechanics 639, https://doi.org/10.1017/S0022112009991522 


\title{
Acceleration, pressure and related quantities in the proximity of the turbulent/non-turbulent interface
}

\author{
MARKUS HOLZNER ${ }^{1,2} \dagger$, B. LÜTHI ${ }^{1,2}$, A. TSINOBER $\mathbf{R}^{1,3,4}$ \\ AND W. KINZELBA CH ${ }^{1,2}$ \\ ${ }^{1}$ International Collaboration for Turbulence Research \\ ${ }^{2}$ Institute of Environmental Engineering, ETH Zurich, Wolfgang-Pauli-Strasse 15, \\ 8093 Zurich, Switzerland
${ }^{3}$ School of Mechanical Engineering, Faculty of Engineering, Tel Aviv University, Tel Aviv 69978, Israel
${ }^{4}$ Institute for Mathematical Sciences and Department of Aeronautics, Imperial College, London SW7 2AZ, UK

(Received 25 February 2009; revised 4 August 2009; accepted 8 August 2009; first published online 23 October 2009)

This paper presents an analysis of flow properties in the proximity of the turbulent/ non-turbulent interface (TNTI), with particular focus on the acceleration of fluid particles, pressure and related small scale quantities such as enstrophy, $\omega^{2}=\omega_{i} \omega_{i}$, and strain, $s^{2}=s_{i j} s_{i j}$. The emphasis is on the qualitative differences between turbulent, intermediate and non-turbulent flow regions, emanating from the solenoidal nature of the turbulent region, the irrotational character of the non-turbulent region and the mixed nature of the intermediate region in between. The results are obtained from a particle tracking experiment and direct numerical simulations (DNS) of a temporally developing flow without mean shear. The analysis reveals that turbulence influences its neighbouring ambient flow in three different ways depending on the distance to the TNTI: (i) pressure has the longest range of influence into the ambient region and in the far region non-local effects dominate. This is felt on the level of velocity as irrotational fluctuations, on the level of acceleration as local change of velocity due to pressure gradients, $\mathrm{D} \boldsymbol{u} / \mathrm{D} t \simeq \partial \boldsymbol{u} / \partial t \simeq-\nabla p / \rho$, and, finally, on the level of strain due to pressureHessian/strain interaction, $(\mathrm{D} / \mathrm{D} t)\left(s^{2} / 2\right) \simeq(\partial / \partial t)\left(s^{2} / 2\right) \simeq-s_{i j} p, i j>0$; (ii) at intermediate distances convective terms (both for acceleration and strain) as well as strain production $-s_{i j} s_{j k} s_{k i}>0$ start to set in. Comparison of the results at Taylor-based Reynolds numbers $R e_{\lambda}=50$ and $R e_{\lambda}=110$ suggests that the distances to the far or intermediate regions scale with the Taylor microscale $\lambda$ or the Kolmogorov length scale $\eta$ of the flow, rather than with an integral length scale; (iii) in the close proximity of the TNTI the velocity field loses its purely irrotational character as viscous effects lead to a sharp increase of enstrophy and enstrophy-related terms. Convective terms show a positive peak reflecting previous findings that in the laboratory frame of reference the interface moves locally with a velocity comparable to the fluid velocity fluctuations.

\section{Introduction}

Many flows observed in nature are partly turbulent (e.g. Scorer 1997), where the turbulent regions are separated from surrounding irrotational (non-turbulent) 
regions by a sharp interface, the so-called turbulent/non-turbulent interface (TNTI). Common examples are smoke plumes from chimneys, effluents from pollution outlets, clouds, volcanic eruptions, seafloor hydrothermal vents and many others. Turbulent entrainment is the process of continuous transition from laminar to turbulent flow across the TNTI. Entrainment has a direct impact on the dynamics and mixing, for example, transport rates of active quantities across the entrainment interface (e.g. momentum, vorticity) as well as passive quantities (e.g. heat). Despite the importance of the problem, there are many open questions that significantly hamper fundamental understanding, accurate modelling and proper parametrization (e.g. see the recent review of Hunt, Eames \& Westerweel 2006). Early experimental and theoretical studies (e.g. Corrsin 1943; Corrsin \& Kistler 1954) discovered several salient features of the spreading of turbulence into regions of undisturbed fluid. In these studies, the TNTI was described as a sharp and strongly convoluted boundary, which fluctuates about a mean position with a velocity that is comparable to the velocity of the energy containing eddies $\mathrm{v}^{\prime}$, where $\mathrm{v}^{\prime}=\left(\left\langle u^{2}\right\rangle+\left\langle v^{2}\right\rangle+\left\langle w^{2}\right\rangle\right)^{1 / 2}(u, v$ and $w$ are the three velocity components in $x_{1}, x_{2}$ and $x_{3}$ directions, $x_{2}$ is the direction normal to the average TNTI and the angle brackets denote the average). On the other hand, a mechanism related to the small scales of the flow was pointed out. Namely, viscous forces were inferred to play a central role for the propagation of the TNTI relative to the fluid. This propagation velocity was assumed to be proportional to the velocity of the viscous eddies, the Kolmogorov velocity $u_{\eta}$. Nevertheless, the well-known observation that the global entrainment rate is independent of the smallscale parameters of the flow (see Tritton 1988; Tsinober 2001; Hunt et al. 2006 and references therein) suggests that the two ranges of scales must be strongly coupled, but it is not known exactly how. On the 'non-turbulent' side of the boundary, the movements of the TNTI were found to induce irrotational velocity fluctuations. The irrotational field can be described by a potential $\Phi$, and the field is specified in a given volume once boundary conditions are assigned. In a theoretical study, Phillips (1955) used a random distribution of normal velocity fluctuations $v_{0}$ at the location of the TNTI, $\hat{x}_{2}=0$, and secondly the condition $\nabla \Phi=0$ at $\hat{x}_{2} \rightarrow \infty$, where $\hat{x}_{2}$ is the distance to the TNTI. With these conditions Phillips predicted that on average the square root of the energy of irrotational velocity fluctuations should decay as $\langle\mathrm{v}\rangle \propto \hat{x}_{2}^{-2}$. A similar, somewhat simpler analysis can be found in Landau \& Lifshitz (1959). The problem of determining $v_{0}$ for a true turbulent flow was not considered by Phillips (1955) and therefore, very close to the TNTI the prediction does not hold. In fact, in the boundary layer experiments of Bradshaw (1967) the prediction was verified, but only for distances larger than several boundary layer thicknesses away from the TNTI. At distances comparable to or smaller than the wavelength carrying most of the turbulent energy (say, an integral scale $L$ ), the behaviour changes due to the influence of turbulence.

One of the goals of the present contribution is to provide a clearer view of processes occurring in the irrotational region in the proximity of the TNTI. The focus is on quantities related to both small scales (e.g. vorticity) and larger scales of motion (e.g. velocity). Till recently, studies of this kind were difficult to conduct experimentally, since they require detailed information on the fields of vorticity and strain. Also for this reason little is known about the processes at small scales in the proximity of the entrainment interface. A few exceptions are recent particle image velocimetry (PIV) and planar laser-induced fluorescence (PLIF) experiments by Westerweel et al. (2002, 2005) of a jet and the particle tracking experiments and simulations by Holzner et al. (2006, 2007, 2008). Direct numerical simulation (DNS) was used to analyse a 
temporally developing wake by Bisset, Hunt \& Rogers (2002) and an axisymmetric jet configuration was studied by Mathew \& Basu (2002). Holzner et al. (2007, 2008) showed that strain decays more slowly than vorticity with distance to the TNTI. However, many aspects, among them the precise reason for this different behaviour or the role of pressure and acceleration, remained open. The present work attempts to elucidate some of these issues and extends the analysis of Holzner et al. (2007) to the irrotational ambient region of the flow. We use particle tracking velocimetry (PTV) data and DNS to discuss the terms of the Navier-Stokes equation,

$$
\frac{\mathrm{D} \boldsymbol{u}}{\mathrm{D} t}=\boldsymbol{a}=-\nabla p / \rho+v \nabla^{2} \boldsymbol{u},
$$

and the balance equation for enstrophy, written as

$$
\frac{\mathrm{D}}{\mathrm{D} t} \frac{\omega^{2}}{2}=\omega_{i} \omega_{j} s_{i j}+\nu \omega_{i} \nabla^{2} \omega_{i}
$$

and rate of strain, reading

$$
\frac{\mathrm{D}}{\mathrm{D} t} \frac{s^{2}}{2}=-s_{i j} s_{j k} s_{k i}-\frac{1}{4} \omega_{i} \omega_{j} s_{i j}-s_{i j} \frac{\partial^{2} p}{\partial x_{i} \partial x_{j}}+v s_{i j} \nabla^{2} s_{i j} .
$$

In addition, we consider also the decomposition of the material derivative in its Eulerian parts, i.e. $\mathrm{D} / \mathrm{D} t=\partial / \partial t+\boldsymbol{u} \cdot \nabla$. In the next section we describe the methods that were used in this study, before we discuss the results in $\S 3$ and finally draw our conclusions.

\section{Method}

Both the experiment and simulation were also described in previous studies by the authors and for the details the reader is referred to Holzner et al. (2008) and references therein. Here we summarize the main parameters and report small changes in the experiment and processing of experimental data. For the experiment, a planar grid installed horizontally on top of a water tank was used to force the turbulent flow. As the grid oscillation is started, a turbulent region develops. The turbulence then propagates in time along the direction normal to the grid through entrainment of surrounding, initially still fluid. The grid is a fine woven screen with a mesh size of $4 \mathrm{~mm}$ and a diameter of the circular grid bars of $1 \mathrm{~mm}$ and oscillates at a frequency of $6 \mathrm{~Hz}$ and with an amplitude of $5 \mathrm{~mm}$. The global Reynolds number associated with these parameters is of the order of $10^{3}$ (Voropayev \& Fernando 1996). For the measurement of the velocity and velocity derivatives we used the scanning method of three-dimensional PTV described in detail in Hoyer et al. (2005). The method is based on stereoscopic imaging of neutrally buoyant tracer particles seeded into the water. Four Microtron high-speed cameras were operated at the frequency of $500 \mathrm{~Hz}$ and the volume scan rate was $50 \mathrm{~Hz}$. The number of tracked particles per frame was about $5 \cdot 10^{3}$ in a volume of $3 \times 3.2 \times 2.5 \mathrm{~cm}^{3}$, about $2 \mathrm{~cm}$ away from the grid and with a mean interparticle distance of about $1.5 \mathrm{~mm}$. Measurements of particle positions are affected by noise and the signal was low-pass filtered to access quantities like acceleration (see Holzner et al. 2008 and references therein). The velocities and their derivatives were calculated in the same way as described in Hoyer et al. (2005) and Holzner et al. (2007) and they were interpolated on a regular Eulerian grid with $2 \mathrm{~mm}$ spacing. We estimated the Kolmogorov length scale $\eta=0.6 \mathrm{~mm}$ using $\eta=\left(v^{3} / \epsilon\right)^{1 / 4}$, where $\epsilon=2 v\left\langle s^{2}\right\rangle$ is the measured mean dissipation $\left(s^{2}=s_{i j} s_{i j}\right.$ is the rate of strain, $s_{i j}$ 
are the components of the rate of strain tensor and $v$ is the kinematic viscosity of the fluid). The Taylor microscale $\lambda$ is estimated at $7 \mathrm{~mm}$ and the Kolmogorov time scale is $\tau_{\eta}=0.3$ s. The r.m.s velocity of the turbulent flow $\mathrm{v}^{\prime}$ is about $8 \mathrm{~mm} \mathrm{~s}^{-1}$ and the Taylor microscale Reynolds number was $R e_{\lambda}=50$. Since the interparticle distance is almost a factor three larger than $\eta$, the experiment is spatially under-resolved. This means that the conclusions to be drawn will be to some extend qualitative and this is precisely one of the reasons why results from both experiment and numerical simulations will be presented. The accuracy of the experimental technique was assessed by Lüthi, Tsinober \& Kinzelbach (2005); see also the validations in Gulitski et al. (2006), where velocity gradients measured via hot-wire techniques were compared to PTV measurements and the results in Schumacher, Sreenivasan \& Yakhot (2007), which indicate that under-resolved data reproduces faithfully the flow at scales about two times smaller than those resolved, at least as far as the instantaneous dissipation rate is concerned. Since our measurements are concerned with a rather different flow situation compared to those cited above (e.g. involving sharp interfaces), we performed an additional check: we produced a 'synthetic' experimental data set with the use of Lagrangian data from DNS (see Holzner et al. 2008 for details on the algorithms used for the integration of particle trajectories). The trajectories were adapted to the real experiment in terms of coarser spatial and temporal resolution and Gaussian position noise was added to mimic instrument noise. The trajectories were processed by using the PTV algorithms and gradients of the velocity, $\partial u_{i} / \partial x_{j}$, and acceleration, $\partial a_{i} / \partial x_{j}$, were compared pointwise to the accurate (i.e. well resolved) values. With this method we obtained relative errors on the order of $10 \%$ and $22 \%$ for derivatives of the velocity and acceleration, respectively, in agreement with the estimates in Lüthi et al. (2005). It was also verified that the experimental error did not increase after quantities were interpolated onto an Eulerian grid. The processing was extended to allow also for measurements of the pressure gradient $\nabla p$. The pressure gradient was obtained indirectly from (1.1) in the form $-\nabla p / \rho=\boldsymbol{a}-\nu \nabla^{2} \boldsymbol{u}$ by using the measurements of Lagrangian acceleration $\boldsymbol{a}$, and the viscous term $\nu \nabla^{2} u$, i.e. involving second derivatives of the velocity. The pressure-strain and the viscous term in (1.3) can presently not be obtained through PTV, but they are available from the DNS. DNS were performed at two different Reynolds numbers in a box of fluid initially at rest (Holzner et al. 2008). Random (in space and time) velocity perturbations are applied at the boundary $x_{2}=0$. The method of boundary velocity assignment determines the velocity scale, $V=\max \left(V_{i}\right)$ and the length scale $\Delta_{l}$. Together with the viscosity of a fluid $v$ these parameters define the Reynolds number $R e=V \Delta_{l} / \nu=1000$ and 4000 of the simulations. The corresponding Taylor microscale Reynolds numbers are $R e_{\lambda}=50$ and 110, respectively. The Navier-Stokes equations are solved with a finite differences scheme and with time advancement computed by a semi-implicit Runge-Kutta method (Nikitin 2006). The resolution is $256 \times 256 \times 256$ grid points in $x_{1}, x_{2}$ and $x_{3}$ direction. The size of the domain is a box of side lengths $5 L, 3 L, 5 L$ for the lower and $2.5 L, 1.5 L, 2.5 L$ for the higher Reynolds number, respectively. The local Kolmogorov length scale is about twice the spacing between grid points for both simulations and the ratios $\lambda / \eta$ are about 12 and 20 for $\operatorname{Re}_{\lambda}=50$ and 110 , respectively. The flow is inhomogeneous in $x_{2}$ direction and therefore also the following direction-dependent parameters were calculated: the estimates of the surrogates $\eta_{i}=15 v\left\langle s_{i i}^{2}\right\rangle$ lie within the range $\eta \pm 4 \%$, the values of $\lambda_{i}=\left(\left\langle u_{i}^{2}\right\rangle /\left\langle s_{i i}^{2}\right\rangle\right)^{1 / 2}$ are within $\left(\lambda_{1}+\lambda_{2}+\lambda_{3}\right) / 3 \pm 9 \%$ and the fluctuating r.m.s. velocities for the three directions are estimated at $v^{\prime} / \sqrt{3} \pm 14 \%$ for both experiment and simulations (no summation over repeated indices applies here). 

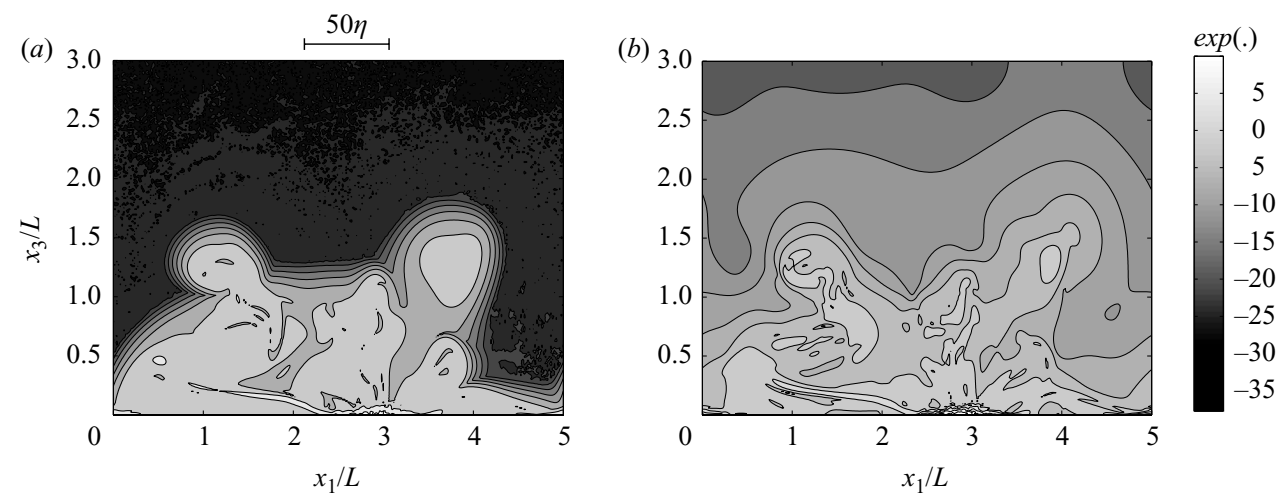

FIGURE 1. Logarithmic contour levels of $\omega^{2} \tau_{\eta}^{2}(a)$ and $s^{2} \tau_{\eta}^{2}(b)$ from a DNS $R e_{\lambda}=50$ snapshot at $x_{3}=2.5 \mathrm{~L}$. The colour bar refers to both $(a)$ and $(b)$.

\section{Results}

We analyse the spreading of a turbulent flow into its irrotational ambient. In both experiment and simulation the forcing is located in the plane $x_{2}=0$ and turbulence spreads on average along $x_{2}>0$. As illustrative example, figure 1 shows logarithmic contour plots of the quantities $\omega^{2}$ (figure $1 a$ ) and $s^{2}$ (figure $1 b$ ) obtained from a representative DNS $R e_{\lambda}=50$ snapshot. It can be seen (figure $1 a$ ) that there are regions where $\omega^{2}$ is high, with values, say, of the order of $10^{0}$ or higher, bounded by regions where $\omega^{2}$ is many decades lower. On the other hand, the qualitative behaviour of $s^{2}$ is very different (figure $1 b$ ). This quantity also decreases, but much more slowly, as pointed out in Holzner et al. (2007). The following statistical analysis is done with respect to the distance to the TNTI, $\hat{x}_{2}$, defined as $\hat{x}_{2}=x_{2}-x_{2}^{*}$, where $x_{2}^{*}\left(x_{1}, x_{3}, t\right)$ marks the instantaneous three-dimensional position of the TNTI detected by using a fixed threshold on enstrophy (for details see Holzner et al. 2007 and references therein). All quantities are ensemble averaged over homogeneous directions $x_{1}, x_{3}$ and time, i.e. $\langle q\rangle\left(\hat{x}_{2}\right)=\left\langle q\left(x_{1}, \hat{x}_{2}, x_{3}, t\right)\right\rangle_{x_{1}, x_{3}, t}$.

Figure 2 shows the same quantities, $\omega^{2}$ and $2 s^{2}$, conditionally averaged as described above. In this and the following figures, when not explicitly stated otherwise, lines represent results obtained from DNS at $R e_{\lambda}=50$, symbols are from the measurement. As noted by Bisset et al. (2002), Mathew \& Basu (2002), Westerweel et al. (2005), Holzner et al. (2006) and Holzner et al. (2007), for instance, we see that $\omega^{2}$ drops from high values to very low values within a short distance. We remark that this distance (at the considered Reynolds number) is comparable to a few Kolmogorov length scales $\eta$, and the region $\hat{x}_{2} / \eta>5$ is regarded as irrotational. The question whether in general the distance to the irrotational region is proportional to $\eta$ or might rather scale with a different length scale of the flow is addressed below as a final point of this section. Experimentally, it is not possible to obtain quantities like $\omega^{2}$ lower than a level of noise represented on the figure by error bars. In this and the following figures, the error bars represent an r.m.s value of the respective quantity taken over the interval where this noise level is reached. As mentioned before, $2 s^{2}$ decays comparatively slower and the measured points closely follow the line obtained from the simulation until again some noise level is reached. In the turbulent region, the approximate relation $\left\langle\omega^{2}\right\rangle \simeq\left\langle 2 s^{2}\right\rangle$ holds, similar to (statistically stationary) homogeneous turbulence. We recall the convention introduced in Holzner et al. (2007), where three physically distinct regions with respect to the behaviour of the term $\nu \omega_{i} \nabla^{2} \omega_{i}$ were defined (see figure $2 a$ (top)): 

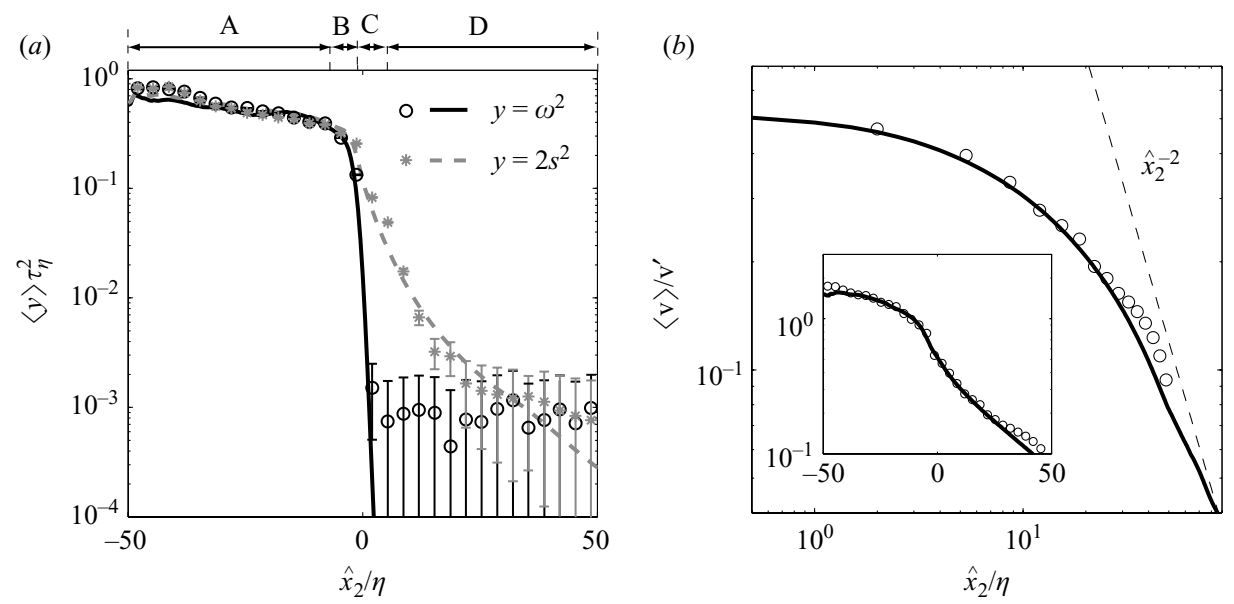

FIGURE 2. Average profiles of $\omega^{2}\left(\hat{x}_{2}\right)$ and $2 s^{2}\left(\hat{x}_{2}\right)$ from PTV (symbols), and DNS at $R e_{\lambda}=50$ (lines) relative to the TNTI, $\hat{x}_{2}=x_{2}-x_{2}^{*}$, on semilogarithmic scale $(a)$. The axes are normalized by using the Kolmogorov length and time scales. The error bars represent the accuracy of the measurement. The symbol $\langle\cdot\rangle$ denotes the ensemble average of the respective quantity. $(b)$ Profile of $\mathrm{v}=\sqrt{u^{2}+v^{2}+w^{2}}$ normalized over the r.m.s. velocity on $\log -\log$ and in the inset on semilogarithmic axes.

(A) the turbulent region, $\hat{x}_{2} / \eta<-1$, in which the behaviour of the viscous term $\nu \omega_{i} \nabla^{2} \omega_{i}$ is 'normal', i.e. it is negative in the mean and $(B, C)$ the intermediate regions $-1<\hat{x}_{2} / \eta<5$, where the viscous term is positive in the mean $\left\langle\nu \omega_{i} \nabla^{2} \omega_{i}\right\rangle>0$, and responsible for the sharp increase of $\omega^{2}$. We introduce a fourth region $(D)$, the interval $\hat{x}_{2} / \eta>5$, denoted as irrotational region. Due to the irrotational nature of this region, the viscous term $\nu \nabla^{2} \boldsymbol{u}$ is vanishing and (1.1) reduces to $\mathrm{D} \boldsymbol{u} / \mathrm{D} t=\boldsymbol{a}=-\nabla p / \rho$ and, since $v \nabla^{2} \boldsymbol{u}$ is zero, also $v \nabla^{2} s_{i j}=0$, (in addition, $\omega_{i} \omega_{j} s_{i j}=0$ ) and hence (1.3) reduces to $(\mathrm{D} / \mathrm{D} t)\left(s^{2} / 2\right)=-s_{i j} s_{j k} s_{k i}-s_{i j}\left(\partial^{2} p / \partial x_{i} \partial x_{j}\right)$. Therefore, in region $D$ the Lagrangian acceleration is irrotational and defined by pressure gradients only and viscosity can not play any role for the change of strain. Phillips (1955) theoretically predicted that the square root of the energy, $\mathrm{v}=\left(u^{2}+v^{2}+w^{2}\right)^{1 / 2}$, of irrotational velocity fluctuations (on average) decrease with distance to the interface as $\hat{x}_{2}^{-2}$. In figure $2(b)$ we show the distance dependence of the magnitude of this quantity and we note that it attenuates, but the predicted slope is not yet reached within the considered span, similar to the results in Bradshaw (1967). We shall see that there is a close connection between the distance dependence of velocity fluctuations and the behaviour of a variety of other quantities analysed in the following.

First, we consider the acceleration of fluid particles and its decompositions. Figure 3 shows the behaviour of the moduli of the total acceleration and its decomposition in pressure gradient and viscous term, (1.1) (figure $3 a$ ), as well as its decomposition in Eulerian components (figure $3 b$ ). The procedure is that first the modulus of a quantity is taken and then it is conditionally averaged in space and time, i.e. $\langle|q|\rangle_{x_{1}, x_{3}, t}$. In homogeneous turbulence, $\boldsymbol{a}$ is known to be driven mostly by pressure gradients and therefore the quantity is mostly irrotational. This is increasingly the case the higher the Reynolds number (e.g. Vedula \& Yeung 1999; Tsinober, Vedula \& Yeung 2001). This trend is confirmed in figure $3(a)$, where the modulus of the viscous term on the turbulent side is (on average) about an order of magnitude smaller than the modulus of the pressure gradient, in agreement with the results in Vedula \& Yeung 

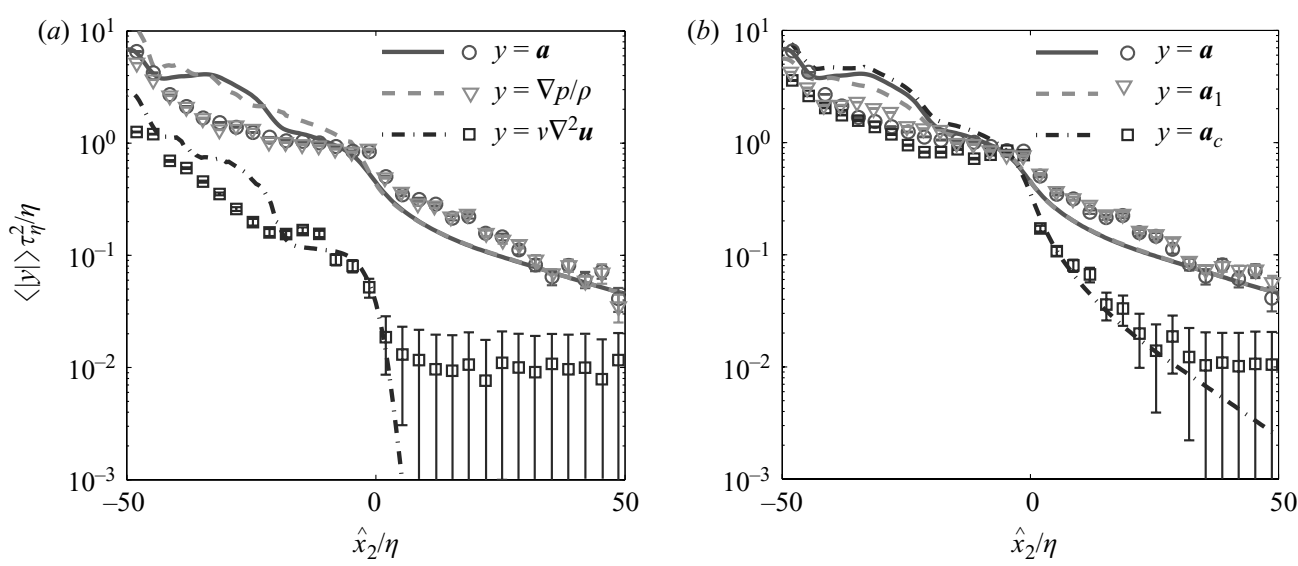

FIGURE 3. (a) Average profiles of the moduli of terms of (1.1) and (b) Lagrangian acceleration and its Eulerian components.

(1999) and Tsinober et al. (2001). Consistent with the above discussion, the term $v \nabla^{2} \boldsymbol{u}$ drops very sharply on the positive $\hat{x}_{2}$-axis and in region $D$ the acceleration is entirely driven by pressure gradients and therefore a purely irrotational quantity. It is noteworthy that a decrease of the Reynolds number in homogeneous turbulence is qualitatively very different from the spatial transition to irrotational flow (with smaller local Reynolds number) considered here. In the former case, the term $v \nabla^{2} \boldsymbol{u}$ gains relative importance for decreasing $R e$, while in the latter case the term $v \nabla^{2} u$ vanishes. Hence, $\boldsymbol{a}$ is irrotational in region $D$, because the velocity field is irrotational, seemingly similar to region $A$, where $\boldsymbol{a}$ is also mostly but not entirely irrotational. However, the irrotationality in region $A$ is due to 'mutual cancellation' of acceleration components, as outlined in the following. The total acceleration $\boldsymbol{a}$ is a sum of local $\boldsymbol{a}_{l}$ and convective parts $\boldsymbol{a}_{c}$, i.e. $\boldsymbol{a}=\boldsymbol{a}_{l}+\boldsymbol{a}_{c}=\partial \boldsymbol{u} / \partial t+\boldsymbol{u} \cdot \nabla \boldsymbol{u}$. In statistically stationary and homogeneous turbulence it is known that typically $\left|\boldsymbol{a}_{l}\right| \sim\left|\boldsymbol{a}_{c}\right| \gg|\boldsymbol{a}|$. The reason is that $\boldsymbol{a}_{l}$ and $\boldsymbol{a}_{c}$ almost mutually cancel each other out, i.e. the two vectors are typically antialigned (e.g. Tsinober et al. 2001; Gulitski et al. 2006). This is increasingly the case the higher the Reynolds number (e.g. Tsinober et al. 2001). In homogeneous turbulence, the local acceleration $\boldsymbol{a}_{l}$ is a purely solenoidal quantity (because the velocity field is solenoidal), whereas the convective acceleration $\boldsymbol{a}_{c}$ has also an irrotational part. The local acceleration cancels out most of the solenoidal part of $\boldsymbol{a}_{c}$, so that the resulting $\boldsymbol{a}$ is in fact mostly irrotational. Due to the relatively low Reynolds number in the present work, we note that all three quantities are comparable in magnitude in region $A$ (see figure $3 b$ ). The figure shows that the modulus of $\boldsymbol{a}_{c}$ drops considerably faster than those of $\boldsymbol{a}$ and $\boldsymbol{a}_{l}$ across the TNTI, and $\boldsymbol{a}$ is more and more close to $\boldsymbol{a}_{l}$ at farther distances. Very different from region $A, \boldsymbol{a}_{l}$ and $\boldsymbol{a}_{c}$ are both irrotational in region $D$, but $\boldsymbol{a}_{c}$ becomes very small compared to $\boldsymbol{a}_{l}$ so that $\boldsymbol{a}$ is very close to $\boldsymbol{a}_{l}$. The faster decay of $\boldsymbol{a}_{c}$ in region $D$ can be partly explained by the fact the the fluctuating velocity attenuates and $\boldsymbol{a}_{c}$ is quadratic in the velocity, whereas $\boldsymbol{a}_{l}$ is linear in the velocity. However, this argument alone is not sufficient, as for a complete explanation also an argument on the behaviour of the derivatives $\partial / \partial t$ and $\partial / \partial x$ is needed and this point remains open.

As a next step we analyse in more detail the dynamical reasons for the different behaviour of enstrophy and strain and analyse all terms in the respective balance equations, (1.2) and (1.3). Figure 4(a) shows the enstrophy terms of (1.2), the total 


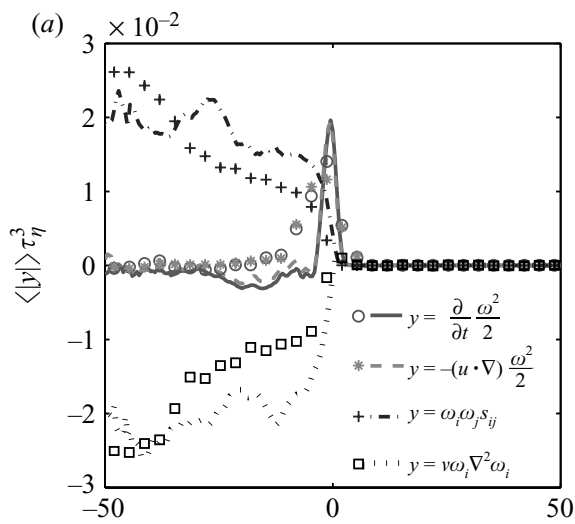

(b)

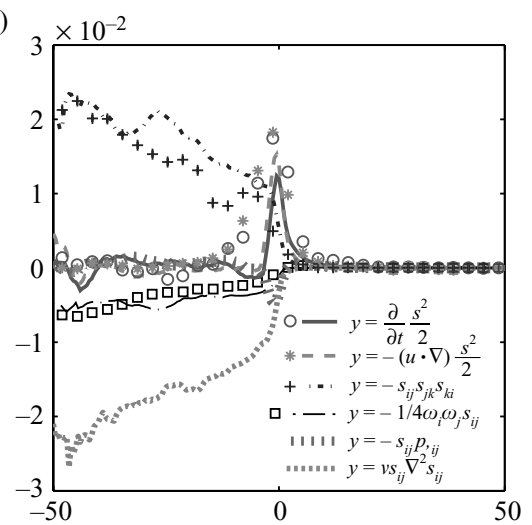

(c)

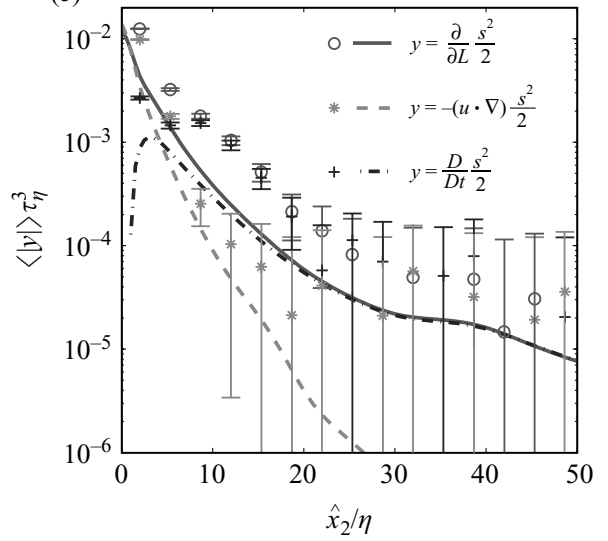

(d)

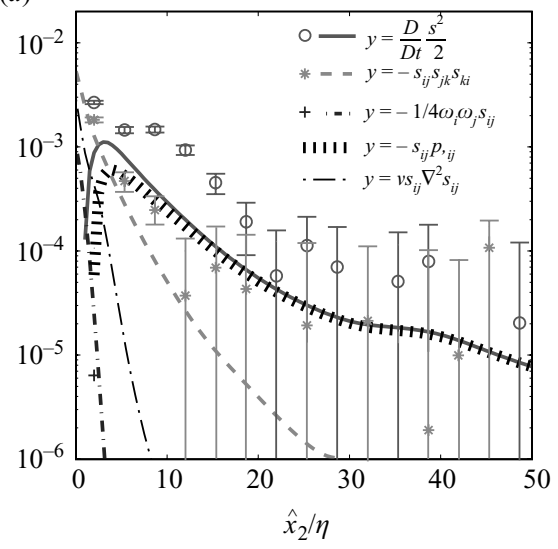

FIGURE 4. Average profiles of the terms of (1.2) (a) and (1.3) (b) and logarithmic profiles of $(\mathrm{D} / \mathrm{D} t)\left(s^{2} / 2\right)$ and its decomposition in Eulerian components $(c)$ and of the terms of $(1.3)(d)$.

change is represented by the two Eulerian components, where the convective term is plotted for convenience with a minus sign. The same is shown in Figgure $4(b)$ for the strain terms of (1.3). On the turbulent side the average production terms are positive and approximately balanced by viscous dissipation, i.e. $\left\langle\omega_{i} \omega_{j} s_{i j}\right\rangle \simeq\left\langle\nu \omega_{i} \nabla^{2} \omega_{i}\right\rangle$ and similarly on figure $4(b)$ we show that $\left\langle s_{i j} s_{j k} s_{k i}\right\rangle+1 / 4\left\langle\omega_{i} \omega_{j} s_{i j}\right\rangle \simeq\left\langle v s_{i j} \nabla^{2} s_{i j}\right\rangle$, as it is known in homogeneous statistically stationary turbulence. Notably, both the average local and convective terms show pronounced positive peaks in the proximity of the origin, whereas on the turbulent side they are small, as they are known to be in homogeneous turbulence. In correspondence to the peak, the magnitudes of the average $(\partial / \partial t)\left(\omega^{2} / 2\right)$ and $-(\boldsymbol{u} \cdot \nabla) \omega^{2} / 2$ are close to each other (and the same is true for the strain terms), whereas the other terms are comparatively small at the origin. This shows that the change of $\omega^{2}$ at a fixed location is mostly determined by the convective transport of the fluid. This result reflects previous observations from point measurements in (statistically stationary) shear flows that the TNTI fluctuates about a mean position and is swept past the probe with a velocity that is close to the turbulent velocity fluctuations of the fluid at that location (e.g. Corrsin \& Kistler 1954). In other words, the local advancement of the TNTI in the laboratory frame of reference is governed by $\mathrm{v}^{\prime}$. This should not be confused with the velocity of the TNTI relative to the fluid, which is governed by a different velocity scale, namely the 

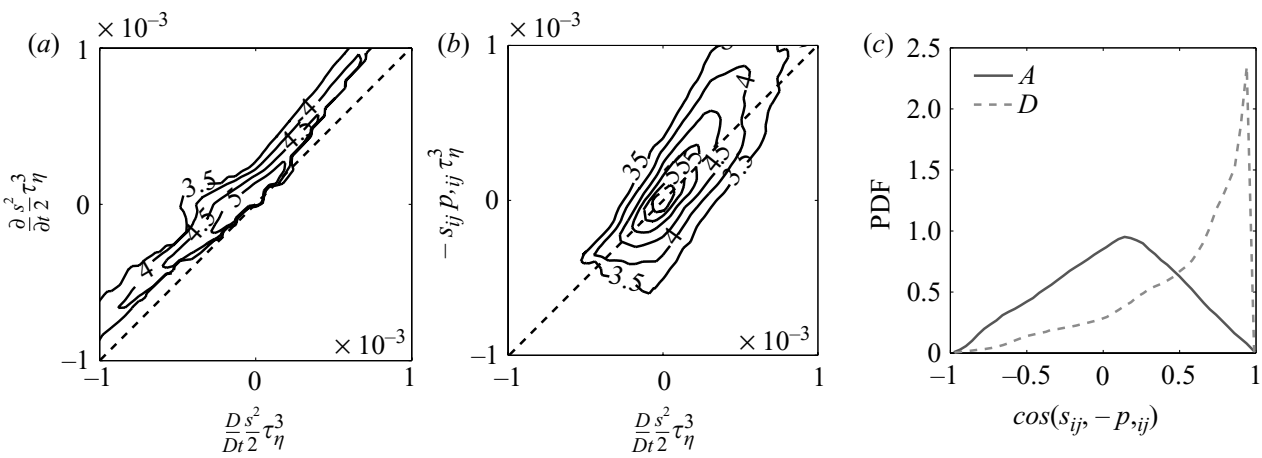

Figure 5. Joint PDF of $(\mathrm{D} / \mathrm{D} t)\left(s^{2} / 2\right)$ versus $(\partial / \partial t)\left(s^{2} / 2\right)(a),(\mathrm{D} / \mathrm{D} t)\left(s^{2} / 2\right)$ versus $-s_{i j} p, i j$ (b) in region $D$ with logarithmic isocontours and PDF of the cosine between $s_{i j}$ and $-p, i j$ for the regions $A$ and $D(c)$, from DNS at $R e_{\lambda}=50$ only.

Kolmogorov velocity $u_{\eta}$ (see Holzner et al. 2008). Similar to the case of enstrophy, also for the local change of strain the convective transport is dominating the dynamics at close distances to the TNTI (figure $4 b$ ), but further away the behaviour changes as will be shown in the following. Figure 4(c) shows the material change of $s^{2}$ and its decomposition in Eulerian components. Similar to the acceleration, the convective term for strain drops faster than its local counterpart, and the material change of $s^{2}$ becomes more and more close to its local derivative. A simple argument to explain in part this behaviour is again that the convective term is cubic in the velocity, while the other two terms are quadratic. Next, we have a closer look at the terms in the equation for strain, (1.3). They are shown in figure $4(d)$, where the terms $\omega_{i} \omega_{j} s_{i j}$ and $v s_{i j} \nabla^{2} s_{i j}$ are plotted with reversed sign to allow for logarithmic representation. We see that pressure has the longest range of influence into the ambient, and that at far distances, $\hat{x}_{2} \sim 50 \eta$ for the considered Reynolds number $R e_{\lambda}=50$, non-local effects dominate the evolution of strain. Also here, the question whether in general the distance to the far region is proportional to $\eta$ or might rather scale with a different length scale is addressed further below. The strain production term $-s_{i j} s_{j k} s_{k i}$ drops faster than $(\mathrm{D} / \mathrm{D} t)\left(s^{2} / 2\right)$ and $-s_{i j} p, i j$, similar to the convective term seen before. The reasons for the different behaviour of $-s_{i j} s_{j k} s_{k i}$ and $-s_{i j} p$, ij lie probably in the non-local nature of $-s_{i j} p,{ }_{i j}$ as contrasted to the local nature of $-s_{i j} s_{j k} s_{k i}$.

Figures 5(a) and 5(b) show joint PDFs of the terms $(\mathrm{D} / \mathrm{D} t)\left(s^{2} / 2\right)$ versus $(\partial / \partial t)\left(s^{2} / 2\right)$ and $(\mathrm{D} / \mathrm{D} t)\left(s^{2} / 2\right)$ versus $-s_{i j} p_{i j}$, respectively, for the region $D$. We find that the contours are almost aligned with the bisector (dashed line) and they are (in figure $5 b$ more strongly than figure $5 a$ ) skewed towards the positive axes. Hence we show that even pointwise there is an approximate balance between $(\mathrm{D} / \mathrm{D} t)\left(s^{2} / 2\right)$ and $(\partial / \partial t)\left(s^{2} / 2\right)$ and $(\mathrm{D} / \mathrm{D} t)\left(s^{2} / 2\right) \simeq-s_{i j} p,{ }_{i j}>0$, i.e. pressure Hessian/strain interaction is clearly the governing mechanism for the amplification of strain in region $D$. The low magnitude of these normalized quantities in region $D\left(\sim 10^{-3}\right)$ indicates that there might be a more appropriate normalization factor than $\tau_{\eta}^{3}$ for these quantities in the irrotational region. The non-local process in the irrotational region is also manifested in strong geometrical effects. We define the cosine of the angle between the strain tensor and pressure Hessian as $\cos \left(s_{i j}, p, i j\right)=I \cdot I I\left\{I^{2}\right\}^{-1 / 2}\left\{I I^{2}\right\}^{-1 / 2}$, where $I=s_{i j}$ and $I I=-p, i j$. The Probability Density Function (PDF) of this quantity is shown in figure 5(c) for the regions $A$ and $D$ and we note that the PDF is strongly positively skewed in region $D$, in contrast to region $A$, where it is more symmetric. Figure 6 shows PDFs of the cosine 

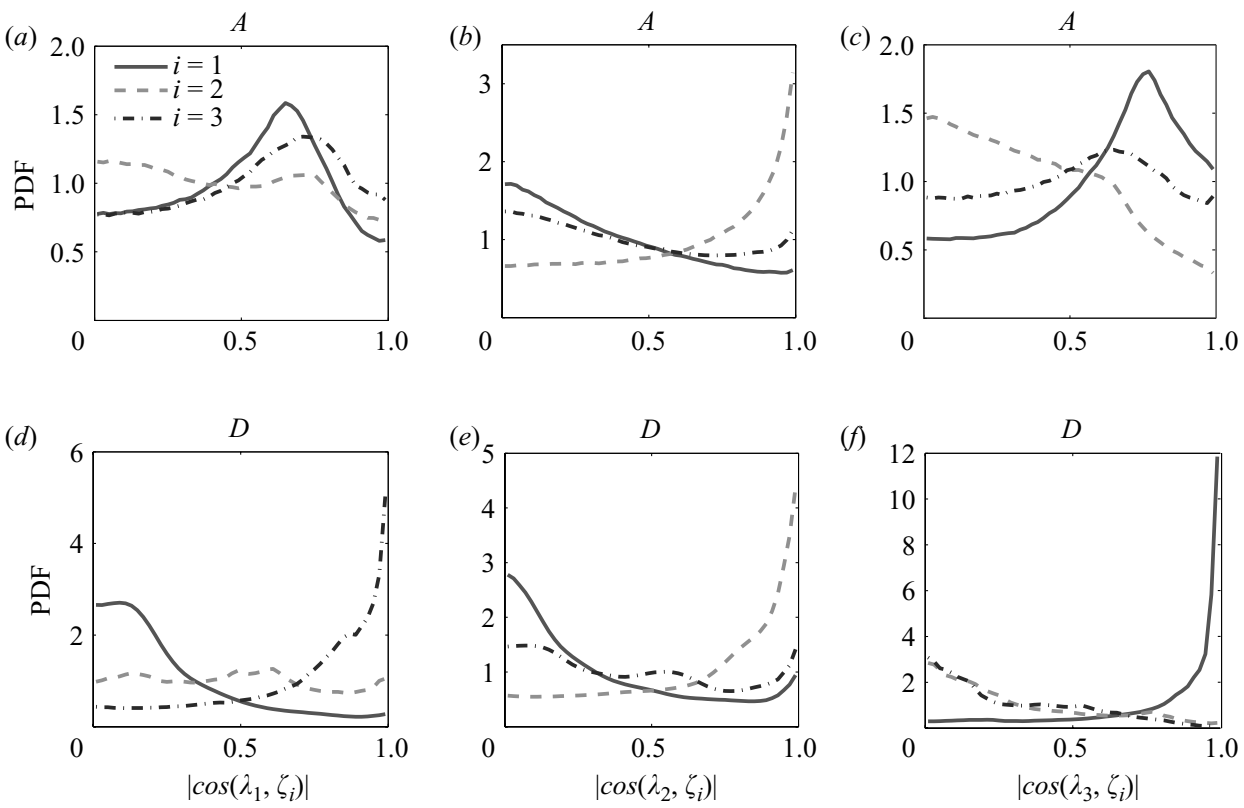

FIGURE 6. PDF of the absolute values of the cosines of the angle between the three eigenvectors of the rate of strain tensor $\lambda_{i}$ and the pressure Hessian $\zeta_{i}$ respectively for the regions $A(a-c)$ and $D(d-f)$, from DNS at $R e_{\lambda}=50$ only.

between the three eigenvectors of the rate of strain tensor $\lambda_{i}$ and the pressure Hessian $\zeta_{i}$ respectively for the turbulent region (figure $6 a-c$ ) and the irrotational region (figure $6 d-f$ ). For the turbulent region (figure $6 a-c$ ) the results are in close agreement to the ones of Kalelkar (2006) obtained from DNS of decaying isotropic turbulence. We find a preferential alignment angle between $\left(\lambda_{1}, \zeta_{1}\right)$ of about $\pi / 4$, whereas the intermediate eigenvector $\lambda_{2}$ is predominantly aligned with $\zeta_{2}$. The compressive eigenvector $\lambda_{3}$ has a preferential alignment angle with $\zeta_{3}$ again close to $\pi / 4$, whereas it is preferably not aligned with $\zeta_{2}$. In the irrotational region, the eigenvectors $\zeta_{1}$ and $\zeta_{3}$ are found to be predominantly aligned with $\lambda_{3}$ and $\lambda_{1}$, respectively. On the other hand, the behaviour of the second eigenvector is qualitatively the same as in the turbulent region.

Finally, figure 7 compares the distance dependence of quantities as obtained from DNS at $R e_{\lambda}=50$ (thick lines) and $R e_{\lambda}=110$ (thin lines), where the distance is normalized by $L$ (figure $7 a, d, g$ ), $\lambda$ (figure $7 b, e, h$ ) and $\eta$ (figure $7 c, f, i$ ). Figure $7(a-c$ ) compares the behaviour of $\omega^{2}$ and $2 s^{2}$, where the error bars represent the numerical noise level of the quantity $\omega^{2}$. This level is of the order of $10^{-10}$ and decreases somewhat with distance to the TNTI. The sharper bend of $\omega^{2}$ at $x_{2} / \eta \sim 5$ (figure $7 c$ ) for the higher Reynolds number case is due to a lower variance of the conditionally averaged quantity at that distance. In figure 7(c) the two curves showing $\omega^{2}$ collapse and the curves corresponding to $2 s^{2}$ show the same slope in region $D$, whereas the curves in figure $7(b)$ are overall closer to each other. Similarly, also for the terms of (1.1) (figure $7 d-f$ ) and terms of (1.3) (figure $7 g-i$ ), the normalization by $\eta$ yields approximately the same slopes of the curves in region $D$ for the two Reynolds numbers. On the other hand, some curves are closer to each other when $\hat{x}_{2}$ is normalized by $\lambda$, also if their slopes in region $D$ are different (e.g. figure $7 h$ ). We can therefore rule out $L$ as a scaling parameter, but it is not fully clear from the results at these moderate Reynolds numbers whether $\eta$ or $\lambda$ is the appropriate parameter 


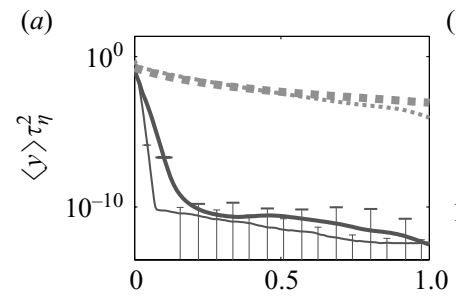

(b)

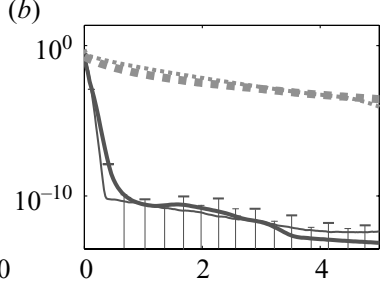

(c)
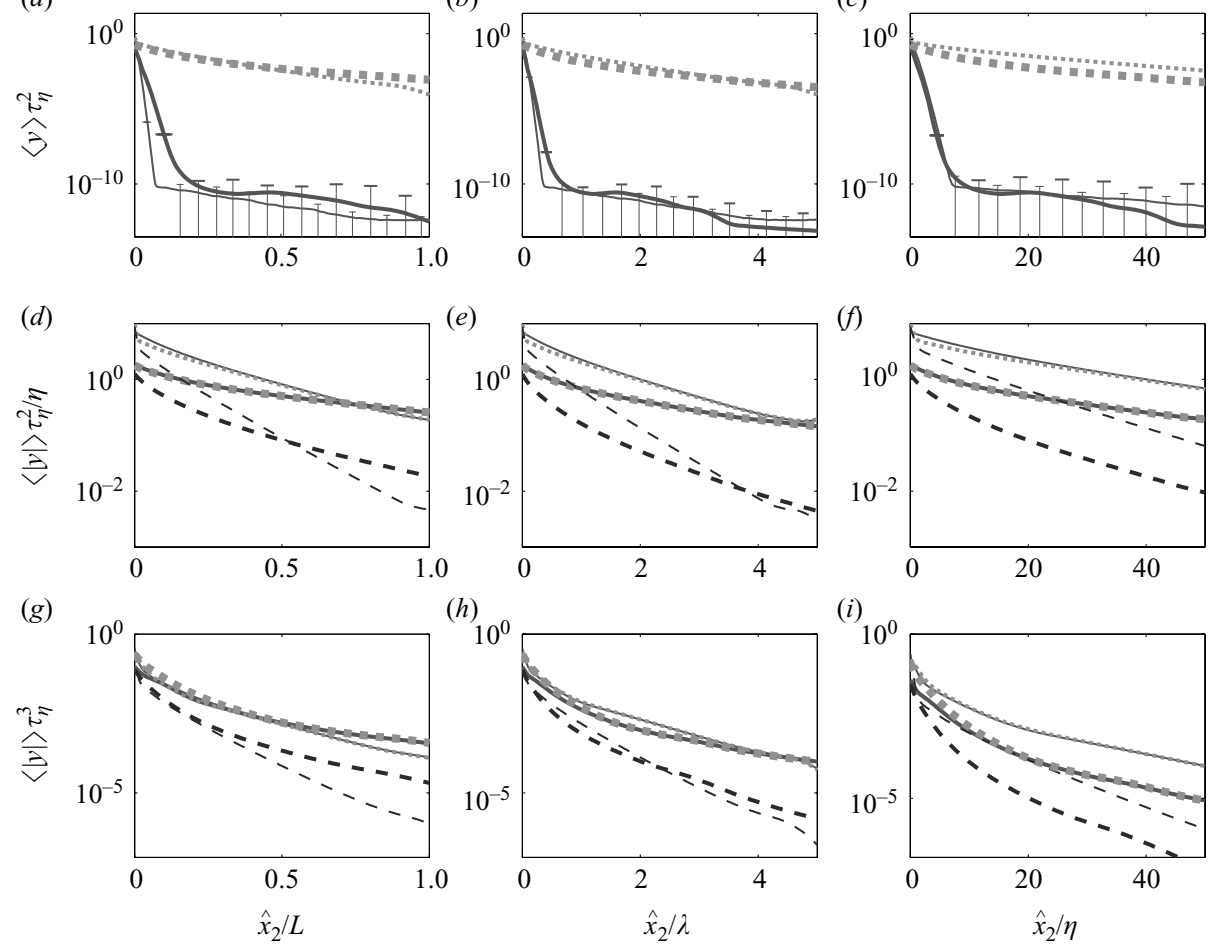

(h)
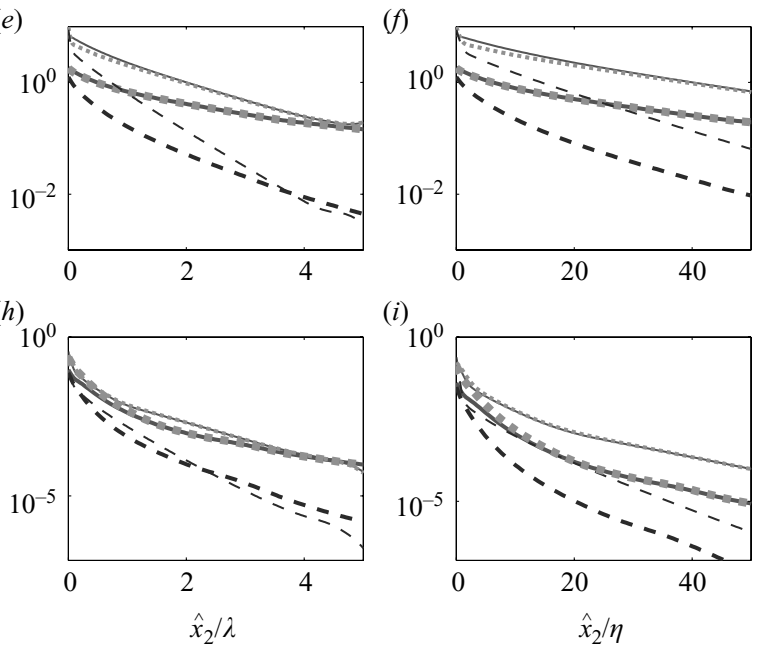

FIGURE 7. Distance dependence of quantities from DNS $R e_{\lambda}=50$ (thick lines) and DNS $R e_{\lambda}=110$ (thin lines), where $\hat{x}_{2}$ is normalized by $L(a, d, g), \lambda(b, e, h)$ and $\eta(c, f, i) .-y=\omega^{2}$, $\cdots y=2 s^{2}(a-c) ;-y=\boldsymbol{a}, \cdots y=\boldsymbol{a}_{l},-y=\boldsymbol{a}_{c}(d-f) ;-y=(\mathrm{D} / \mathrm{D} t)\left(s^{2} / 2\right), \cdots y=-s_{i j} p, i j, \cdots$ $y=-s_{i j} s_{j k} s_{k i}(g-i)$.

to characterize the distance to the irrotational or far region. Future measurements at much higher Reynolds numbers will have to answer whether the appropriate scaling of $\hat{x}_{2}$ and the quantities themselves is possibly 'mixed' or rather based on inner variables only.

\section{Conclusions}

In summary, we used a particle tracking experiment and DNS to examine various flow properties in the proximity of the TNTI. From the technical point we note that the qualitative agreement between measurements and simulation is satisfactory, except when the magnitudes of the measured quantities are smaller than the accuracy of the experimental method, since the numerical noise level lies far below the experimental one. We analysed quantities like the velocity and the acceleration of fluid particles and small-scale quantities like enstrophy and strain. The evolution of all these quantities in the nominally non-turbulent ambient flow was shown to be governed by the turbulent region non-locally through pressure. In particular, pressure gradients are solely responsible for the acceleration of fluid particles and pressure gradients induce deformation of fluid elements through pressure-strain interaction. These effects dominate at larger distances from the interface. The second way turbulence influences the ambient flow is through convective acceleration and strain terms. They become increasingly important closer to the TNTI, where the streamlines are presumably 
more contorted by the complex shape of the interface. Comparison of the results from two simulations at $R e_{\lambda}=50$ and 110 suggested that either the Kolmogorov length scale or Taylor microscale of the flow is the appropriate measure to delimit the distance to the irrotational or far regions, rather than an integral length scale, but experiments and simulations at higher Reynolds will be needed to further clarify this point. The dominance of convective terms in the close proximity of the TNTI explains why the local fluctuations of the position of the TNTI in the laboratory frame of reference are governed by the fluid velocity fluctuations.

\section{REFERENCES}

Bisset, D. K., Hunt, J. C. R. \& Rogers, M. M. 2002 The turbulent/non-turbulent interface bounding a far wake. J. Fluid Mech. 451, 383-410.

BRADSHAW, P. 1967 Irrotational fluctuations near a turbulent boundary layer. J. Fluid Mech. 27 (2), 209-230.

Corrsin, S. 1943 Investigation of flow in an axially symmetric heated jet in air. ACR 3L23 and Wartime Rept W94. NACA.

Corrsin, S. \& Kistler, A. L. 1954 The free-stream boundaries of turbulent flows. Rep. TN-3133, TR-1244, 1033-1064. NACA.

Gulitski, G., Kholmyansky, M., Kinzelbach, W., LÜthi, B., Tsinober, A. \& Yorish, S. 2006 Velocity and temperature derivatives in high Reynolds number turbulent flows in the atmospheric surface layer. Part II. Accelerations and related matters. J. Fluid Mech. 589, 83102 .

Holzner, M., Liberzon, A., Guala, M., Tsinober, A. \& Kinzelbach, W. 2006 Generalized detection of a turbulent front generated by an oscillating grid. Exp. Fluids. 41 (5), 711719.

Holzner, M., Liberzon, A., Nikitin, N., Kinzelbach, W. \& Tsinober, A. 2007 Small scale aspects of flows in proximity of the turbulent/non-turbulent interface. Phys. Fluids 19, 071702.

Holzner, M., Liberzon, A., Nikitin, N., LÜthi, B., KinZelbach, W. \& Tsinober, A. 2008 A Lagrangian investigation of the small scale features of turbulent entrainment through threedimensional-PTV and DNS. J. Fluid Mech. 598, 465-475.

Hoyer, K., Holzner, M., Lüthi, B., Guala, M., Liberzon, A. \& Kinzelbach, W. 2005 Threedimensional scanning particle tracking velocimetry. Exp. Fluids 39 (5), 923-934.

Hunt, J. C. R., Eames, I. \& Westerweel, J. 2006 Mechanics of inhomogeneous turbulence and interfacial layers. J. Fluid Mech. 554, 449-519.

KaLelKar, C. 2006 Statistics of pressure fluctuations in decaying, isotropic turbulence. Phys. Rev. E 73, 046301.

Landau, L. D. \& Lifshitz, E. M. 1959 Fluid Mechanics. Pergamon Press.

LÜTHI, B., Tsinober, A. \& KinzeLbaCh, W. 2005 Lagrangian measurement of vorticity dynamics in turbulent flow. J. Fluid Mech. 528, 87-118.

Mathew, J. \& Basu, A. J. 2002 Some characteristics of entrainment at a cylindrical turbulence boundary. Phys. Fluids 14 (7), 2065-2072.

Nikitin, N. 2006 Finite-difference method for incompressible Navier-Stokes equations in arbitrary orthogonal curvilinear coordinates. J. Comput. Phys. 217 (2), 759-781.

Phillips, O. M. 1955 The irrotational motion outside a free turbulent boundary. Proc. Camb. Phil. Soc. 51, 220-229.

SChumacher, J., SReEnivasan, K. R. \& YaKhot, V. 2007 Asymptotic exponents from low-Reynoldsnumber flows, New J. Phys. 9 (89), 1-19.

SCORER, R. S. 1997 Dynamics of Meteorology and Climate. Wiley.

Tritton, D. J. 1988 Physical Fluid Dynamics, 2nd edn. Clarendon Press.

Tsinober, A. 2001 An Informal Introduction to Turbulence. Springer.

Tsinober, A., Vedula, P. \& Yeung, P. K. 2001 Random Taylor hypothesis and the behaviour of local and convective accelerations in isotropic turbulence. Phys. Fluids 13, 1974-1984. 
Vedula, P. \& Yeung, P. K. 1999 Similarity scaling of acceleration and pressure statistics in numerical simulations of isotropic turbulence. Phys. Fluids 11, 1208-1220.

Voropayev, S. I. \& Fernando, H. J. S. 1996 Propagation of grid turbulence in homogeneous fluids. Phys. Fluids 8 (9), 2435-2440.

Westerweel, J., Fukushima, C., Pedersen, J. M. \& Hunt, J. 2005 Mechanics of the turbulent/nonturbulent interface of a jet. Phys. Rev. Lett. 95, 174501.

Westerweel, J., Hoffmann, T., Fukushima, C. \& Hunt, J. C. R. 2002 The turbulent/non-turbulent interface at the outer boundary of a self-similar turbulent jet. Exp. Fluids 33, 873-878. 